Supporting Information :

\title{
Multiphase engineered BNT-based ceramics with simultaneous high polarization and superior breakdown strength for energy storage applications
}

Chaoqiong Zhu ${ }^{a}$, Ziming Cai ${ }^{b}$, Bingcheng Luo ${ }^{c}, X u$ Cheng $^{a}$, Limin Guo ${ }^{d}$, Ying Jiang ${ }^{d}$ Xiuhua Cao ${ }^{e}$, Zhenxiao Fu ${ }^{e}$, Longtu Li ${ }^{a}$, Xiaohui Wang ${ }^{a, *}$

a State Key Laboratory of New Ceramics and Fine Processing, School of Materials Science and Engineering, Tsinghua University, Beijing 100084, China

${ }^{\mathrm{b}}$ School of Materials Science and Physics, China University of Mining and Technology, Xuzhou 221116, China

${ }^{c}$ Department of Engineering, University of Cambridge, CB30FA Cambridge, United Kingdom

d School of Science, Beijing University of Posts and Telecommunications, Beijing 100876, China

e State Key Laboratory of Advanced Materials and Electronic Components, Guangdong Fenghua Advanced Technology Holding Co., Ltd., Guangdong 526000, China

\section{Corresponding Author}

*Xiaohui Wang, E-mail: wxh@mail.tsinghua.edu.cn 


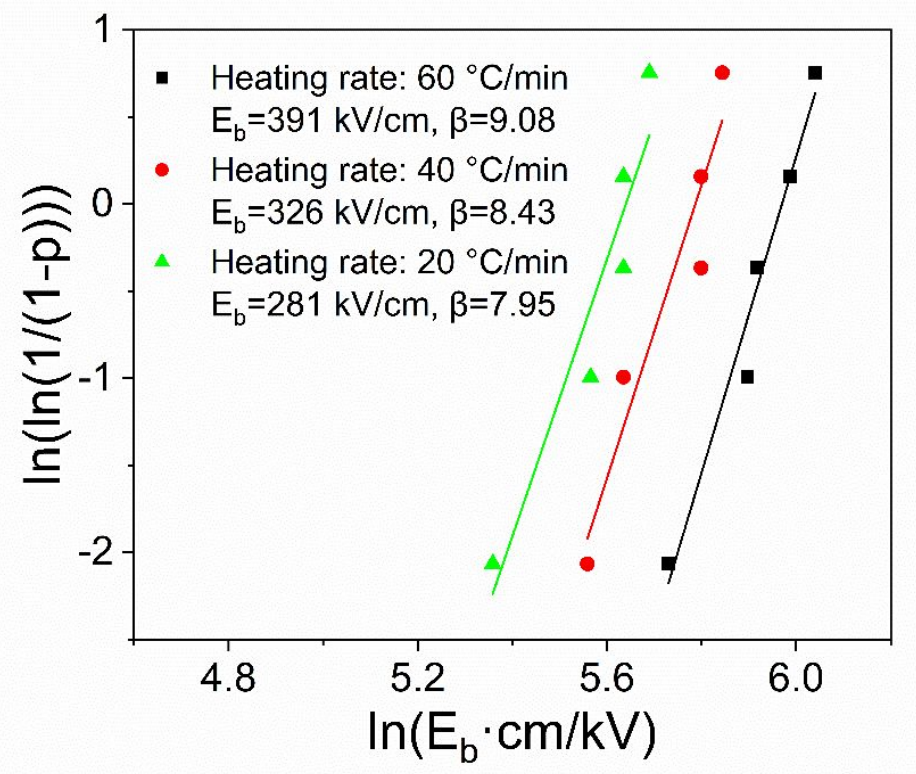

Figure S1. Weibull plots of the DC dielectric breakdown strength for BNTSZNN ceramics with various heating rate.

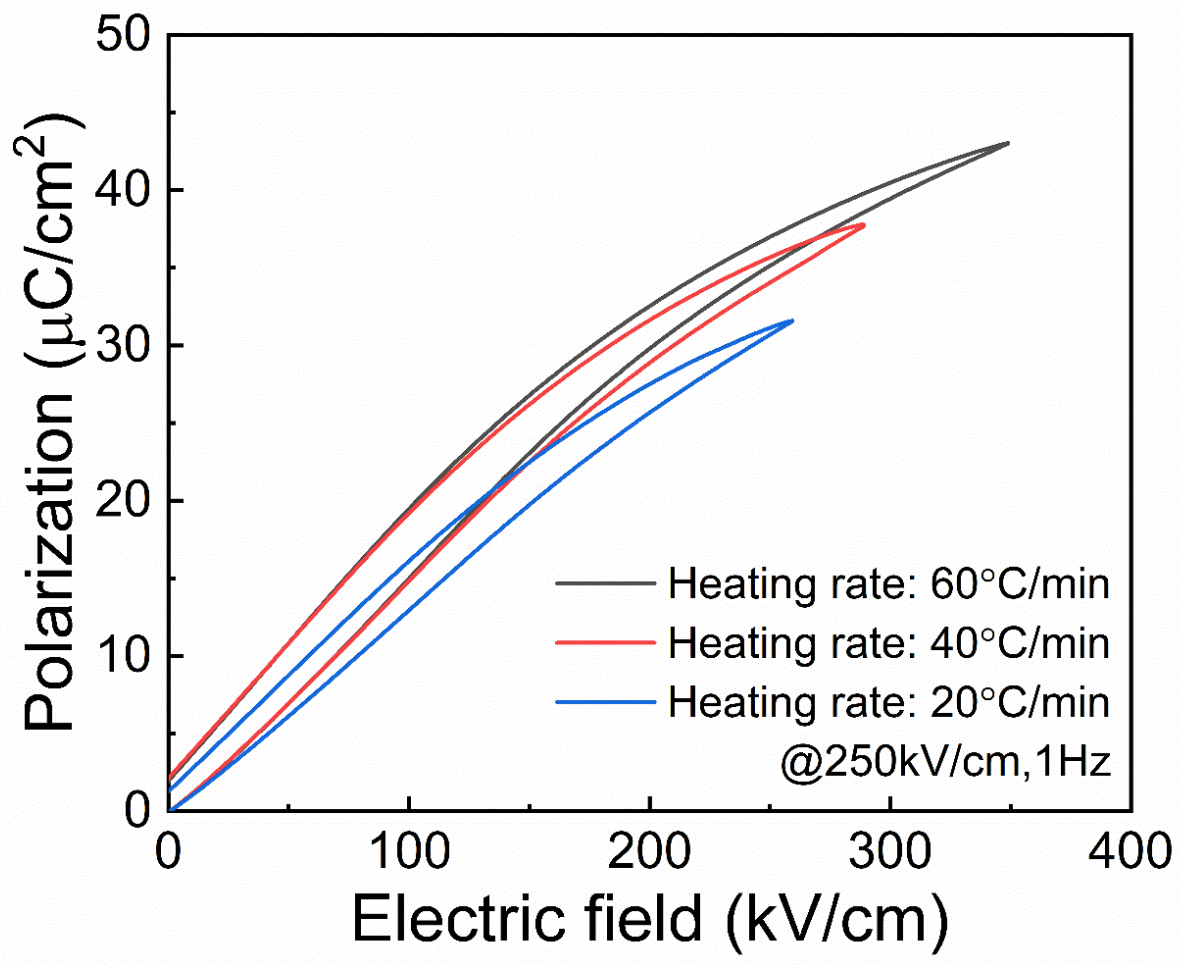

Figure S2. Unipolar hysteresis loops measured under each maximum applied electric field and at $1 \mathrm{~Hz}$ of the BNTSZNN ceramics sintered with various heating rates. 
(a)

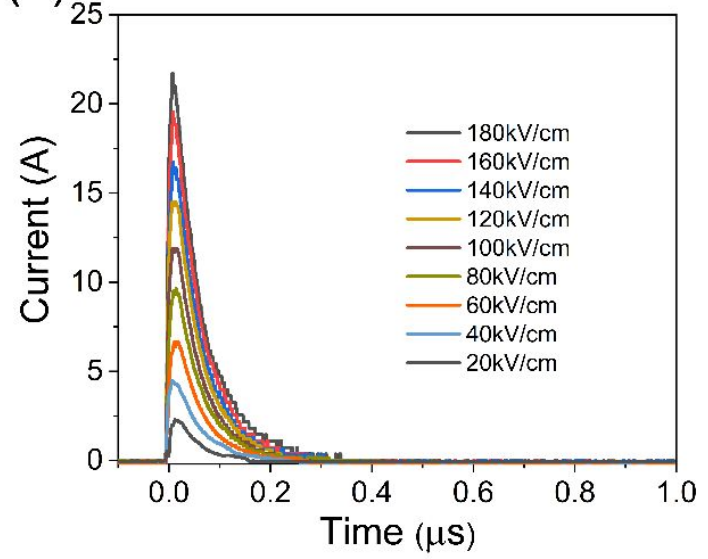

(b)

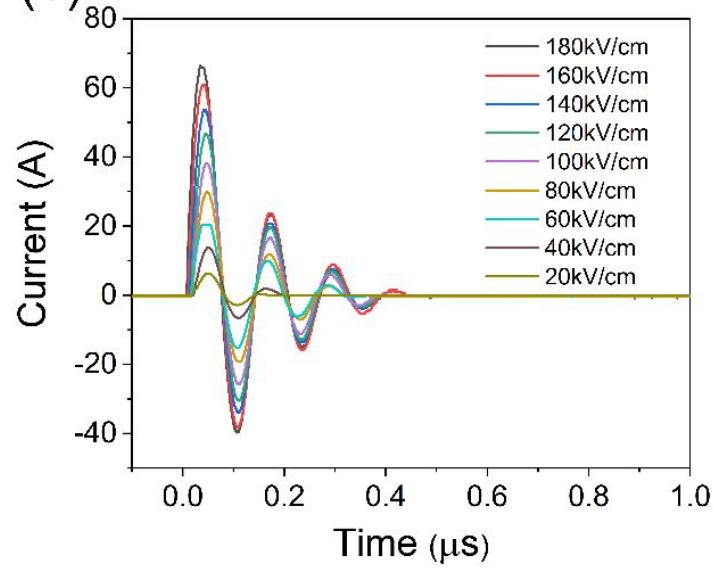

Figure S3. (a) Overdamped and (b) undamped pulsed discharge electric current-time $(I-t)$ curves as a function of the applied electric field for the BNTSZNN-60 sample. 


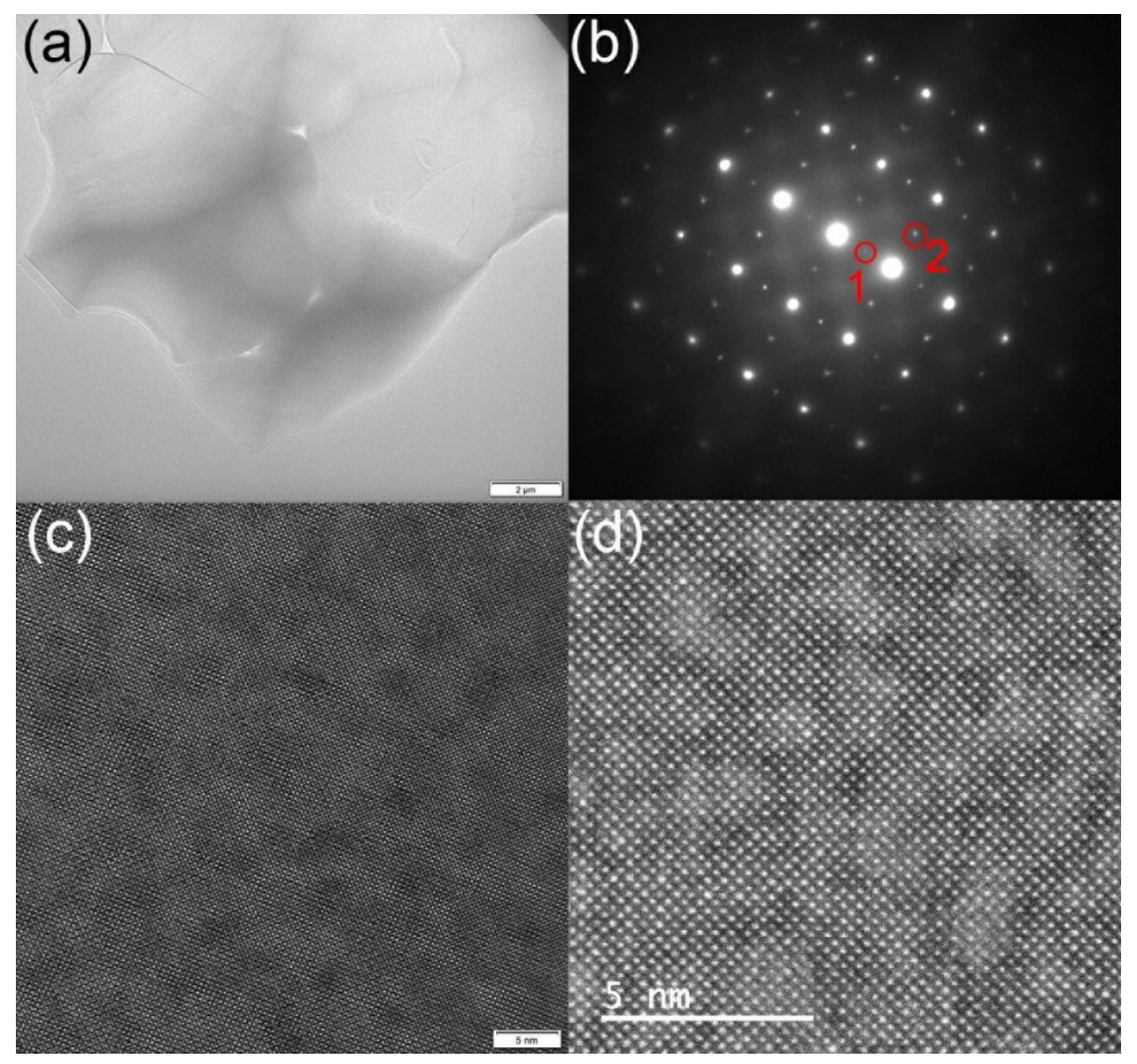

Figure S4. (a) TEM image of BNTSZNN-60. (b) The SEAD pattern along [112]c with superlattice dots for T phase (dot 1) and R phase (dot 2). (c) high resolution TEM and (d) aberration-corrected high resolution TEM image of BNTSZNN-60. 